\title{
Self-pumping effects and radiation linewidth of Josephson flux-flow oscillators
}

\author{
Koshelets, V.P.; Shitov, S.V.; Shchukin, A.V.; Filippenko, L.V.; Mygind, Jesper; Ustinov, A.V.
}

Published in:

Physical Review B

Link to article, DOI:

10.1103/PhysRevB.56.5572

Publication date:

1997

Document Version

Publisher's PDF, also known as Version of record

Link back to DTU Orbit

Citation $(A P A)$ :

Koshelets, V.P., Shitov, S. V., Shchukin, A. V., Filippenko, L. V., Mygind, J., \& Ustinov, A. V. (1997). Selfpumping effects and radiation linewidth of Josephson flux-flow oscillators. Physical Review B, 56(9), 5572-5577. https://doi.org/10.1103/PhysRevB.56.5572

\section{General rights}

Copyright and moral rights for the publications made accessible in the public portal are retained by the authors and/or other copyright owners and it is a condition of accessing publications that users recognise and abide by the legal requirements associated with these rights.

- Users may download and print one copy of any publication from the public portal for the purpose of private study or research.

- You may not further distribute the material or use it for any profit-making activity or commercial gain

- You may freely distribute the URL identifying the publication in the public portal 


\title{
Self-pumping effects and radiation linewidth of Josephson flux-flow oscillators
}

\author{
V. P. Koshelets, S. V. Shitov, A. V. Shchukin, and L. V. Filippenko \\ Institute of Radio Engineering and Electronics RAS, Mokhovaya 11, Moscow 103907, Russia \\ J. Mygind \\ Department of Physics, Technical University of Denmark, B309, DK-2800 Lyngby, Denmark
}

A. V. Ustinov

Physics Institute III, University of Erlangen-Nuremberg, Erwin-Rommel-Strasse 1, D-91058 Erlangen, Germany

(Received 17 March 1997)

\begin{abstract}
Flux-flow oscillators (FFO's) are being developed for integration with a SIS mixer for use in submillimeter wave receivers. The present work contains a detailed experimental study of the dc, microwave, and noise

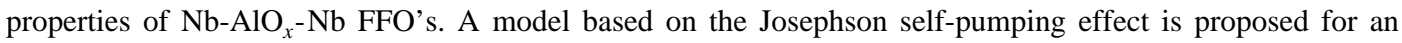
explanation of the experimental current-voltage characteristics. A reliable technique based on harmonic mixing is used to determine the spectral linewidth of the radiation emitted by the integrated FFO's up to $600 \mathrm{GHz}$. Comprehensive measurements of the dependence of the linewidth on the dynamic resistance and the applied magnetic field have been performed. In the resonant regime a linewidth as small as $200 \mathrm{kHz}$ is obtained at 450 GHz. The experimental data are compared with recent theoretical predictions. [S0163-1829(97)02233-9]
\end{abstract}

\section{INTRODUCTION}

Josephson flux-flow oscillators (FFO's) based on the unidirectional and viscous flow of magnetic flux quanta in a long Josephson tunnel junction (LJJ) with relatively high damping ${ }^{1}$ have been successfully employed ${ }^{2-4}$ in fully superconducting-integrated submillimeter-wave receivers. Recently $\mathrm{Nb}-\mathrm{AlO}_{x}-\mathrm{Nb}$ FFO's have been successfully tested above the gap frequency of $\mathrm{Nb}$ (up to $850 \mathrm{GHz}$ ) and power levels sufficient to pump a SIS mixer have been demonstrated (about $5 \mu \mathrm{W}$ at $440 \mathrm{GHz}$ ). Measurements at $500 \mathrm{GHz}$ have given receiver noise temperatures [DSB (double side band)] as low as $140 \mathrm{~K},{ }^{4}$ which is only a few times the fundamental quantum value $h f / k_{B}$ ( $h$ is Planck's constant, and $k_{B}$ is Boltzmann's constant). For spectral radio astronomy applications, besides the low-noise temperature, also a high-frequency resolution of the receiver is very important. This resolution is determined mainly by the linewidth of the local oscillator (LO) and its long-time frequency stability which should be less than $1 \times 10^{-6}$ of the center frequency.

Previous measurements of the FFO linewidth spectrum ${ }^{5-8}$ have demonstrated reasonably low values $[130 \mathrm{kHz}$ at 70 $\mathrm{GHz},{ }^{5}$ about $1 \mathrm{MHz}$ at $280 \mathrm{GHz},{ }^{6,7}$ and $2.1 \mathrm{MHz}$ at $320 \mathrm{GHz}$ (Ref. 8)]. Recently, a simple and reliable technique for linewidth measurements has been developed ${ }^{9,10}$ and FFO linewidths of only a few hundred $\mathrm{kHz}$ can be measured at several hundred $\mathrm{GHz}$. It was found that the narrowest FFO linewidths are realized on the very steep resonant steps in the current-voltage, $(I-V)$ characteristic of the FFO. ${ }^{7,9,10}$ This fine structure superimposed on the flux-flow step (FFS) results in a rather complicated dependence of the FFO linewidth on the applied magnetic field and bias current. Even at the resonant steps the FFO linewidth appears ${ }^{6,7}$ to be almost one order of magnitude larger than predicted by the theory for Josephson oscillations in a lumped tunnel junction ${ }^{11}$ and for fluxon motion in underdamped LJJ's. ${ }^{12}$ The experimental $I-V$ characteristics of the high-current-density FFO's (Refs. 2 and 6) cannot be explained by the existing theoretical models.

Here we report on the results of a detailed experimental study of the dc, microwave, and noise properties of $\mathrm{Nb}-\mathrm{AlO}_{x}-\mathrm{Nb}$ FFO's. The experimental spectral linewidth results are compared with the recent theoretical model by Golubov et al. ${ }^{13}$

\section{EXPERIMENTAL DETAILS}

Long Josephson $\mathrm{Nb}-\mathrm{AlO}_{x}-\mathrm{Nb}$ junctions with overlap geometry are used as FFO's (see Fig. 1). The FFO length $L$ ranges from 450 to $500 \mu \mathrm{m}$, and the width $W$ is about $3 \mu \mathrm{m}$.
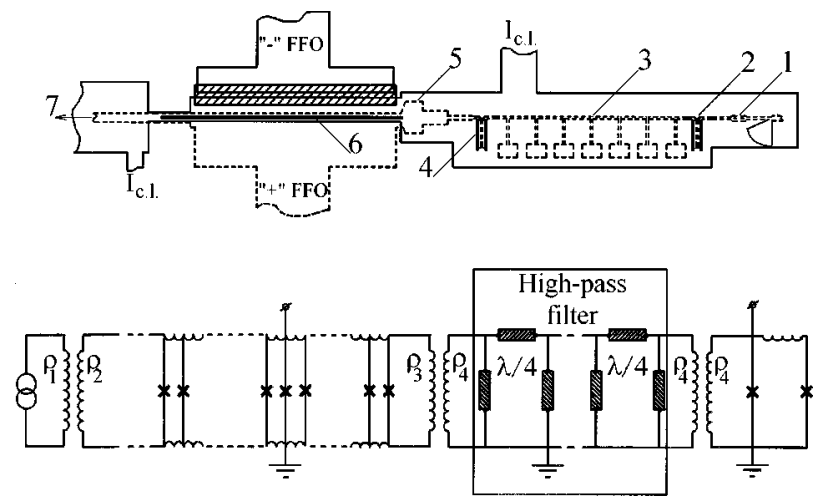

FIG. 1. Layout and simplified equivalent diagram of the test circuit for linewidth measurements. "1,"' SIS mixer; " 2 '” and " 4 ,', dc block; "“3,'” high-pass filter; " 5 ,' Chebyshev impedance matching transformer; "6,", FFO; "7,", connection to the input transformer and fin-line antenna. In the equivalent diagram $\rho_{i}: \rho_{j}$ indicates an impedance transformer. An array of discrete short junctions connected in parallel (marked by $\times$ ) models the long Josephson junction. 


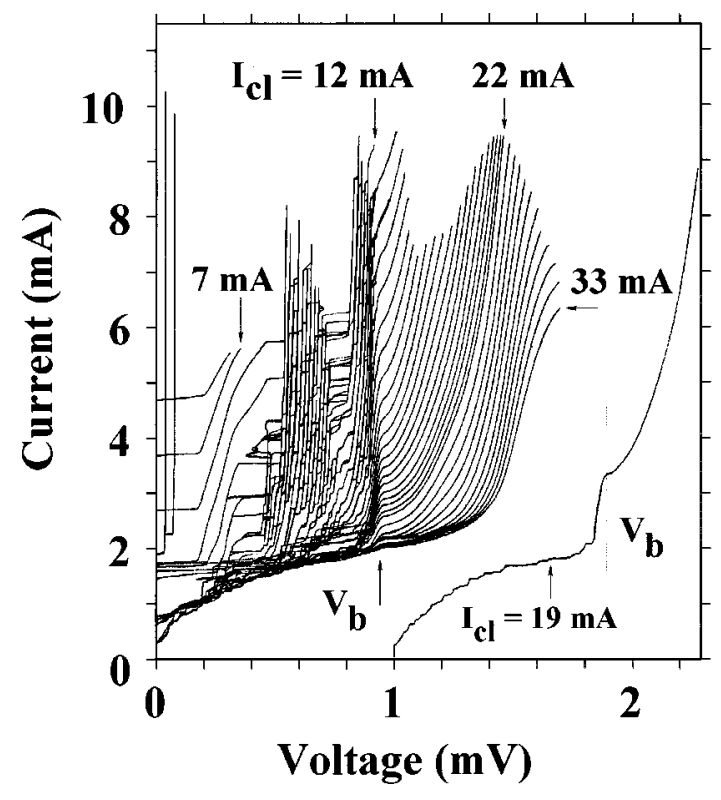

FIG. 2. $I-V$ characteristics of a test FFO recorded at different applied magnetic fields supplied by the control line current $I_{\mathrm{cl}}$. The increment $\Delta I_{\mathrm{cl}}$ is $0.5 \mathrm{~mA}$ for $7 \mathrm{~mA}<I_{\mathrm{cl}}<22 \mathrm{~mA}$, while $\Delta I_{\mathrm{cl}}=1 \mathrm{~mA}$ for $22 \mathrm{~mA}<I_{\mathrm{cl}}<33 \mathrm{~mA}$. The inset (shifted by $1 \mathrm{mV}$ ) shows the definition of the "boundary" voltage $V_{b}$ for one of the $I-V$ characteristics, recorded at $I_{c l}=19 \mathrm{~mA}$.

The value of the critical current density $j_{c}$ is in the range of $2-8 \mathrm{kA} / \mathrm{cm}^{2}\left(\lambda_{J} \approx 8-4 \mu \mathrm{m}\right)$, which corresponds to a specific resistance $R_{n} L W \approx 100-25 \Omega \mu \mathrm{m}^{2}$. The values of the London penetration depth $\left(\lambda_{L} \approx 90 \mathrm{~nm}\right)$ and the junction specific capacitance $\left(C_{s} \approx 0.08 \mathrm{pF} / \mu \mathrm{m}^{2}\right)$ are assumed for the numerical calculations. The FFO's are formed in the window of the $\mathrm{SiO}$ insulator layer between the base and the counterelectrodes. The width $W_{i}$ of the "idle" (overlapping) region, formed by the thicker insulation layer and the overlapping electrodes adjacent (parallel) to the junction, is about the junction width ( $W_{i} \approx 3 \mu \mathrm{m}$ ). The base electrode is employed as a control line to produce the magnetic field $B_{\text {appl }}$ for the FFO. A specially designed integrated circuit comprising the FFO, the SIS mixer, and the high-frequency matching circuits (see Fig. 1) is used for linewidth measurements; the design will be described in Sec. IV. Both the SIS and FFO junctions are fabricated from the same $\mathrm{Nb}-\mathrm{AlO}_{x}-\mathrm{Nb}$ trilayer; details have been described elsewhere. ${ }^{2,4,6,7}$ Additional test FFO's together with the integrated circuits necessary for the FFO linewidth measurement are also placed on the same silicon substrate. The experiments are performed with the samples located inside a cryoperm can and two external $\mu$-metal shields. All electrical connections to the chip are carefully rf shielded and filtered, and the critical components of the room-temperature setup (dc bias, preamplifiers, etc.) are battery powered.

\section{III. dc PROPERTIES OF THE FFO'S}

Typical $I-V$ characteristics of the FFO recorded at a different magnetic fields (produced by the control line current $\left.I_{c 1}\right)$ are shown in Fig. 2. The maximum critical current $I_{c}^{\mathrm{max}}$ is $16 \mathrm{~mA}$, which is about $0.20 \Delta I_{g}$, where $\Delta I_{g}$ is the rise in the quasiparticle current at the gap voltage $V_{g}$. The value of $I_{c}^{\text {max }}$ is suppressed due to strong-coupling effects in the $\mathrm{Nb}$ films (reduction $k=I_{c}^{\mathrm{max}} / \Delta I_{g} \approx 0.72$ for lumped $\mathrm{Nb}-\mathrm{AlO}_{x}-\mathrm{Nb}$ junctions). This value is further reduced because of the nonuniform current distribution in a long junction of overlap geometry. The experimental $I_{c}^{\max }$ corresponds well to the theoretical estimation ${ }^{14} I_{c}^{\max } \approx 2.35 \Delta I_{g} k\left(\lambda_{J} / L\right)^{1 / 2}$. It should be noted that FFO's shaped with an "unbiased overlap extension" used to increase the slope of the FFS (see Ref. 1 and Fig. 1) have a strongly asymmetric magnetic pattern $I_{c}^{\max }\left(B_{\text {appl }}\right)$, and the maximum of $I_{c}^{\max }$ occurs at a nonzero external magnetic field (see Ref. 15).

Zero-field steps (ZFS's) in the $I-V$ characteristics of the FFO were observed at small magnetic fields. The position of the ZFS is dependent on the propagation velocity of the electromagnetic wave along the FFO. The so-called Swihart velocity $c_{\mathrm{Sw}}$ is the maximum velocity of fluxons in the junction. The "idle" superconducting microstrip lines parallel to the junction have a much higher propagation velocity than that of the bare Josephson transmission line. This results in an increase of the effective Swihart velocity $c_{\mathrm{Sw}, \text { eff }}$, and consequently, the voltage of the ZFS, $V_{\mathrm{ZFS}}$, is significantly increased in comparison with the bare junction. The value of $c_{\mathrm{Sw}, \text { eff }}$ depends ${ }^{16}$ on the ratio $W_{i} / W$, and using the parameters stated above we find that $c_{\mathrm{Sw} \text {,eff }}$ is about $1.3 c_{\mathrm{Sw}}$ in our sample design.

The so-called displaced linear slope (DLS) is observed in the $I-V$ characteristics of the FFO at low magnetic fields. With increasing $B_{\text {appl }}$ (see Fig. 2 at $V<400 \mu \mathrm{V}$ ) the DLS branch shifts almost linearly with $B_{\text {appl }}$ towards higher voltages. With the FFO biased in the DLS regime, we found a "smearing" of the $I-V$ characteristics of the SIS detector at $V \approx 0$ and $V \approx V_{g}$ (instead of the usual distinct superposition of quasiparticle and Shapiro steps). It means, most probably, that the FFO, when biased on the DLS, operates rather as a wide-band noise source than as a narrow-band oscillator. Such behavior was reported in a recent paper, ${ }^{17}$ where an extremely broad radiation linewidth was observed for a FFO biased at the DLS. Numerical simulations ${ }^{17}$ showed that the FFO dynamics at DLS is characterized by irregular fluxon oscillations that resemble a chaotic state. Qualitatively, the complicated fluxon dynamic can be attributed to excitation of the internal oscillation modes in the "soft" fluxon chain at weak magnetic fields.

With increasing magnetic field the DLS abruptly transforms into the FFS's, which subsequently splits into a series of resonant Fiske steps (FS's) as clearly seen in Fig. 2. This splitting takes place up to a specific "boundary" voltage $V_{b}$ where the FS's disappear. At the same voltage $V_{b}$, the maximum (switching) current of the FFS abruptly increases. As also seen in Fig. 2 for $V>V_{b}$, the FFS becomes smooth and with increasing magnetic field it persists up to the gap voltage. It should be noted that this "boundary" is typical for all investigated LJJ's with high current density $\left(j_{c}\right.$ $>1 \mathrm{kA} / \mathrm{cm}^{2}$ ). This feature does not depend significantly neither on the exact junction geometry and its dimensions nor on the coupling to the external microwave circuits. For example, the $I-V$ characteristics presented in Fig. 2 are measured for an isolated (without microwave circuit) standard overlap geometry test LJJ without the "unbiased overlap extension." To clarify the definition of $V_{b}$, the $I-V$ character- 


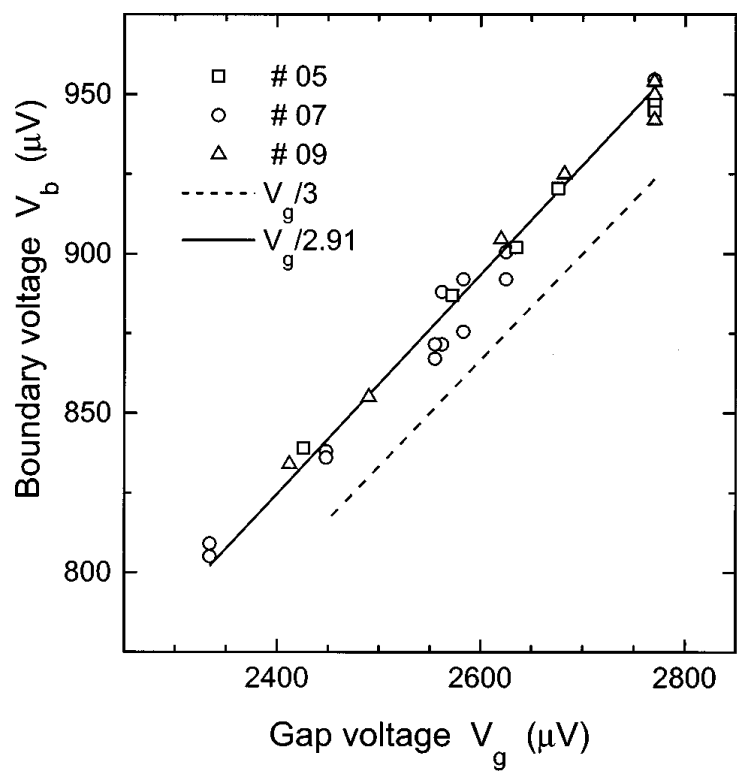

FIG. 3. The dependence of the "boundary" voltage $V_{b}$ on the gap voltage $V_{g}$ for three different FFO's. The data were obtained by varying the temperature.

istics recorded with a control line current of $19 \mathrm{~mA}$ are shown as an inset in Fig. 2. The boundary voltage $V_{b}$ as a function of $V_{g}$ is plotted in Fig. 3 for three different samples. The values of $V_{b}$ at different temperatures (that results in different $V_{g}$ ) are evaluated from data obtained with a digital storage oscilloscope. From Fig. 3 one can see that the $V_{g} / V_{b}$ ratio is about 3 and is almost independent of temperature. A least-mean-squares fit to the experimental values gives a $V_{g} / V_{b}$ ratio equal to 2.91 (solid line). The dashed line is drawn with slope 3.

The "boundary" voltage on the FFO $I-V$ characteristics can be explained by the effect of Josephson self-coupling (JSC), ${ }^{18-20}$ which basically is absorption of ac Josephson radiation energy by the quasiparticles. This leads to the wellknown phenomenon of photon-assisted tunneling. The JSC which can be derived ${ }^{18,19}$ from Werthamer's equation results in current steps at voltages $V$ determined by $e V+n h f=2 \Delta$ $=e V_{g}$, where $h f$ is the photon energy, $n$ is an integer, and $\Delta$ is the superconductor gap energy. Taking into account the Josephson relation $h f=2 \mathrm{eV}$, the voltage position of the JSC current bumps, $V_{\mathrm{JSC}}$, may be expressed as $V_{\mathrm{JSC}}=V_{g} /(2 n$ $+1)$, which gives $V_{\mathrm{JSC}}=V_{g} / 3$ for $n=1$. According to the theory, ${ }^{18,19}$ the JSC effect on the $I-V$ characteristics should be prominent for Josephson tunnel junctions with high critical current density.

We propose here a simplified LJJ model that can be used to simulate the JSC effect. It is assumed that any short section (say, with length $L_{s}$ ) of the long junction is equally subjected to the JSC. The modeled $I-V$ characteristics for a lumped SIS junction can be used for each section to numerically simulate the JSC effect on the $I-V$ characteristics of the FFO. The calculations were done with the parameters $L_{s}$ $=L / 100, V_{g}=2.77 \mathrm{mV}$, gap smearing $\delta V_{g}=0.2 \mathrm{mV}$, normal state resistance $R_{n}=7 \Omega$, and subgap to normal state resistance ratio $R_{j} / R_{n}=25$. The modeled curve [see Fig. 4(a), solid line] accounts for the main features of the experimental $I-V$ characteristics. The $I-V$ characteristics of the
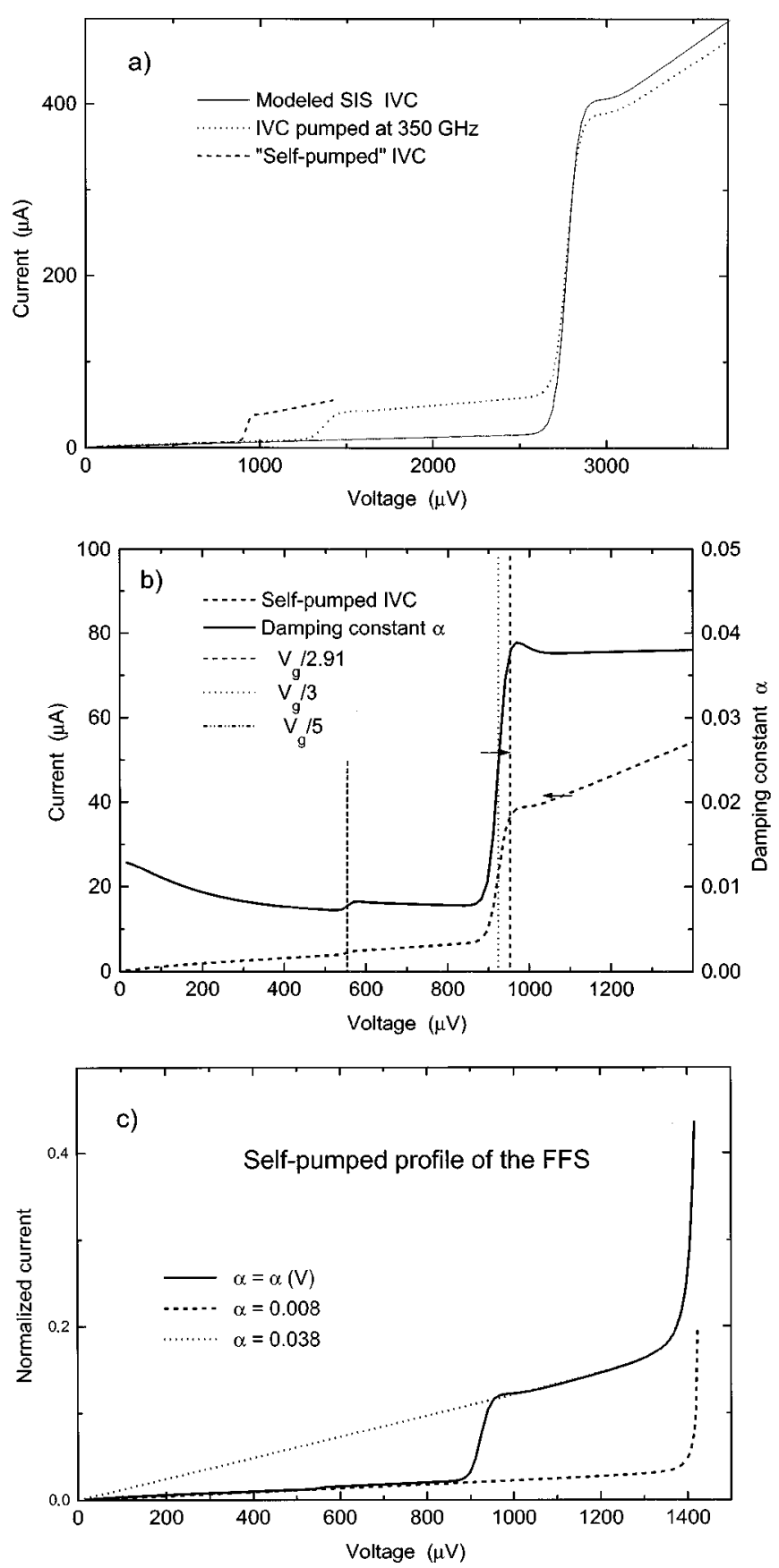

FIG. 4. (a) Modeled SIS $I-V$ characteristics: autonomous (solid line), and pumped at $f=350 \mathrm{GHz}$ (dotted line). The "self-pumped", $I-V$ characteristics are shown by the dashed curve. (b) "Selfpumped" $I-V$ characteristics (dashed line) and damping constant $\alpha_{k}$ (solid curve); see text. (c) Calculated profile of the FFS for the voltage-dependent damping $\alpha_{k}\left(V_{k}\right)$, solid curve. The FFS profiles at fixed $\alpha$ values 0.008 and 0.038 are shown by dotted and dashed lines, respectively.

pumped SIS junction are calculated using the well-known Tien-Gordon expression. An example of the $I-V$ characteristics of the SIS junction pumped at $f=350 \mathrm{GHz}$ is shown in Fig. 4(a) as the dotted line. It is calculated with a normalized rf voltage amplitude $V_{\mathrm{rf}}=0.6 \mathrm{hf} / \mathrm{e}$.

The dashed line in Fig. 4(a) shows the self-pumped $I-V$ characteristics calculated for the voltage range $0<V_{k}$ $<V_{g} / 2$. Since each voltage $V_{k}$ corresponds to a particular 
oscillation frequency $f_{k}=2 e V_{k} / h$, the values of the dc currents $I_{k}^{\mathrm{SP}}\left(V_{k}\right)$ are calculated as the photon-assisted current of the junction pumped at $f_{k}$. The normalized amplitude of the rf voltage is taken to be constant $\left(e V_{\mathrm{rf}}\right) /\left(h f_{k}\right)=0.6$, which corresponds to a rf amplitude of about $1.2 \mathrm{mV}$ at $500 \mathrm{GHz}$ $\left(V_{k} \approx 1 \mathrm{mV}\right)$. This value is in good agreement with the experimental estimations. ${ }^{4}$ In a real FFO, $V_{\text {rf }}$ depends on the FFO bias current ${ }^{2,4}$ and the dc voltage. This dependence (as well as the influence of the junction capacitance) is neglected here in order to keep our qualitative calculation simple. Similar calculations were performed in Ref. 21 though no self-pumped $I-V$ characteristics were reconstructed.

An enlarged view of the modeled "self-pumped" $I-V$ characteristics $I_{k}^{\mathrm{SP}}\left(V_{k}\right)$ is shown in Fig. 4(b) as the dashed curve. The corresponding values of the damping constant $\alpha_{k}$ are presented by the solid curve. The damping constant is calculated as

$$
\alpha_{k}=\left(\beta_{k}^{c}\right)^{-1 / 2},
$$

with

$$
\beta_{k}^{c}=\left(2 \pi / \Phi_{0}\right) I_{c}^{\max }\left(R_{j k}\right)^{2} C,
$$

where $\Phi_{0}=h / 2 e$ is the magnetic flux quantum and $C$ is the junction capacitance. Both the damping constant $\alpha_{k}$ and the McCumber parameter $\beta_{k}^{c}$ are calculated in every $k$ th point using the resistance $\left(R_{j}\right)_{k}=V_{k} / I_{k}^{\mathrm{SP}}$. One can see from Fig. 4(b) that the predicted JSC current bumps appear at $V$ $\approx V_{g} /(2 n+1)$ as expected. Furthermore, for $n=1$ the exact value of the sharp boundary is 2.91 , in excellent agreement with the experimental value.

The effect of self-pumping explains not only the FFO current bumps at $V \approx V_{g} / 3$ as discussed above, but also the abrupt vanishing of the FS's. The latter effect may be ascribed to the increase of the damping from 0.008 to 0.038 at the voltage $V \approx V_{g} / 3$ [see Fig. 4(b)]. The geometric resonances (FS's) only exist for low normalized damping $\alpha l$ $<1$, where $l=L / \lambda_{J}$ is the junction length normalized to the Josephson penetration length. If the specific damping is sufficiently low (say, $\alpha \leqslant 0.01$ ), this condition can be satisfied even for large normalized junction lengths, $l=L / \lambda_{J} \geqslant 60$. The FS's smear out when the damping increases to a value of about $\alpha l \geqslant 2$. This happens at $V_{g} / 3 \approx V>950 \mu \mathrm{V}$, where the FFO enters the "real" flux-flow regime.

The obtained values of the damping constant $\alpha_{k}$ have been used in the calculations of the FFS profile according to the Marcus-Imry perturbation approach. ${ }^{22}$ The resulting "self-pumped" FFS is shown in Fig. 4(c) by the solid line, which quite resembles the experimental $I-V$ characteristics (see inset of Fig. 2). The FFS's calculated for the fixed $\alpha$ values of 0.008 and 0.038 without the self-pumping effect are shown in Fig. 4(c) by the dashed and dotted lines, respectively. The Marcus-Imry model ${ }^{22}$ does not take into account the surface losses in superconducting electrodes, which are expected to be responsible for the "bending" of the experimental $I-V$ characteristics at higher voltages. We note that in the range of high voltages an accurate $I-V$ characteristics simulation should be based on the microscopic tunneling theory. ${ }^{23}$ One should also take into account the following factors: (i) the spatial distribution of the rf voltage $V_{\mathrm{rf}}$ along the junction, (ii) the dependence of the $V_{\text {rf }}$ on the FFO bias current and dc voltage, and (iii) the frequencydependent dispersion and the surface losses in the junction electrodes. Nevertheless, we find that the simplified approach presented above is rather useful for a qualitative interpretation of the experimental data.

\section{FFO LINEWIDTH}

In order to measure the FFO linewidth in a wide frequency range, we have developed a simple and reliable experimental technique. ${ }^{9,10}$ In principle, this method is suitable for evaluation of any oscillator operated in conjunction with a SIS mixer. Due to its strong nonlinearity, the SIS junction can be employed as a high-number harmonic mixer in which the signal under investigation beats with $m$ th harmonic of a synthesized reference signal.

The layout and a simplified equivalent diagram of the integrated test circuit used for wide-band FFO linewidth measurements $\mathrm{s}^{9,10}$ is shown in Fig. 1. The experimental circuit comprises a twin-junction SIS mixer with tuned-out capacitance " 1 " (SIS junction area $S \approx 1-1.5 \mu \mathrm{m}^{2}$ ), threestage impedance matching transformer " 5 ," and the long Josephson junction (FFO) " 6 " as the device under test. Both the impedance transformer and the capacitance compensation circuit are designed for a center frequency of $450 \mathrm{GHz}$. The mm-wave signal coming from the FFO is mixed in the SIS detector with the $m$ th harmonic of the external reference oscillator to generate a signal at the intermediate frequency $f_{\mathrm{IF}}= \pm\left(f_{\mathrm{FFO}}-m f_{\mathrm{ref}}\right)$. In order to prevent the external oscillator signal (as well as its most powerful low-frequency harmonics) from reaching the FFO, a high-pass microstrip filter " 3 " is employed (the calculated cutoff frequency is about $200 \mathrm{GHz}$ ). Two dc blocks " 2 ", and " 4 "' are inserted in the microstrip line to prevent shortening of the SIS mixer and the FFO at dc and at $f_{\mathrm{IF}}$.

The experimental method has been employed to measure the FFO linewidth in a wide frequency range from 200 up to $580 \mathrm{GHz}$. Using a fairly low-frequency reference oscillator $\left(f_{\text {ref }} \approx 10 \mathrm{GHz}\right)$ with a wide tuning range multiplied to a high harmonic number $(m=20-60)$ enables us to measure the FFO linewidth at any desirable frequency in the given frequency range. Alternatively, working with a high-frequency reference oscillator (say, a Gunn oscillator with $f_{\text {ref }}=70 \mathrm{GHz}, m=3-7$ ) with relatively narrow frequency tuning range would limit the linewidth measurements to a few $\mathrm{GHz}$ wide band around the harmonic voltages $V$ $=\Phi_{0} m f_{\text {ref }}$. In order to perform accurate linewidth measurement $(\Delta f<1 \mathrm{MHz})$, the IF spectra have to be averaged with a sufficiently small video bandwidth $(\sim 1 \mathrm{kHz})$. Due to the high tuning rate $(\delta f / \delta I \sim 5 \mathrm{MHz} / \mu \mathrm{A})$, even a relatively small drift of the control line and/or dc bias currents will result in a significant smearing of the averaged linewidth. We used the source locking microwave counter (measuring the IF frequency $\approx 1.5 \mathrm{GHz}$ ) for frequency locking of the FFO to the $10-\mathrm{GHz}$ synthesiser. ${ }^{9,10}$ The analog output (locking signal) from the microwave counter is used for the fine adjustment of the FFO frequency by tuning the FFO dc magnetic field (via control line current) or its bias current to compensate the IF drift. In both cases frequency locking of the FFO has been successfully realized. The feedback loop was operated at a relatively low frequency of about $1 \mathrm{kHz}$, 


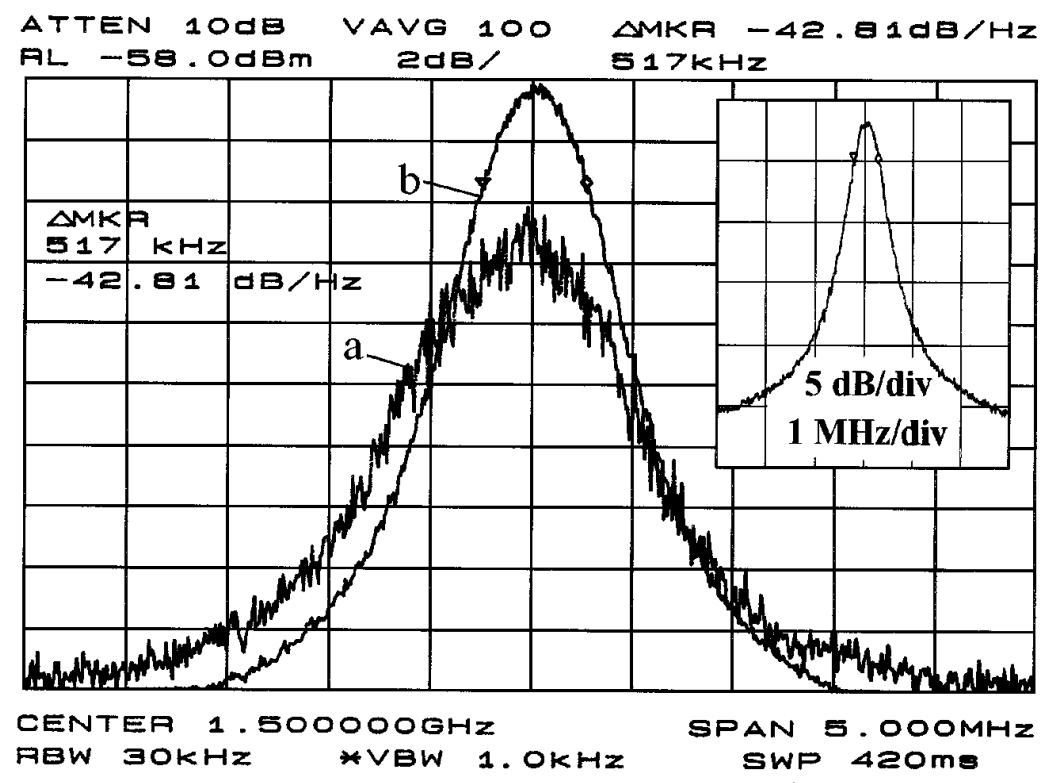

FIG. 5. IF power spectra recorded at $T$ $=4.2 \mathrm{~K}$ when the signal from the FFO $\left(f_{\mathrm{FFO}}\right.$ $=435 \mathrm{GHz})$ is mixed with the 45th harmonic of the synthesizer signal $\left(f_{\text {ref }}=9.7 \mathrm{GHz}\right)$ for the case of (a) an autonomous FFO and (b) the FFO frequency locked by the source locking microwave counter. All spectrum analyzer settings are the same for both curves, except the curve (a) which was measured with a video bandwidth (VBW) of $10 \mathrm{kHz}$, sweep time (SWP) of $50 \mathrm{~ms}$, and averaged 35 times. The inset shows the IF power spectrum (b) recorded with a $5 \mathrm{~dB} / \mathrm{div}$ scale.

actually limited by the low-pass filters in the bias supplies.

The FFO spectra measured at $\mathrm{IF}=1.5 \mathrm{GHz}$ with this technique for both (a) autonomous and (b) frequency-locked FFO's are shown in Fig. 5. The inset in Fig. 5 shows the IF spectra for the frequency-locked FFO (see curve $b$ ) recorded with the vertical scale of $5 \mathrm{~dB} / \mathrm{div}$. The linewidth of the frequency-locked FFO was stable and very reproducible. In fact, it was possible to average the IF spectra for about $1 \mathrm{~h}$ without a noticeable change in the linewidth value. A linewidth $\Delta f$ (full width at half power level) as low as $200 \mathrm{kHz}$ has been measured at $450 \mathrm{GHz}$ at the temperature $T=2 \mathrm{~K}^{9,10}$ It should be noted that the narrowest FFO linewidth is found on the resonant steps (FS's) with extremely low dynamic resistance.

The linewidth $\Delta f$ of the FFO's was measured in different regimes. The experimental data for one of the tested samples are shown in the Fig. 6 as a function of the dynamic resistance $R_{d}=d V / d I$ of the $I-V$ characteristics. The data obtained in the "resonant" regime (at $V<950 \mu \mathrm{V})$ for different runs are shown by circles, while the data for $V$ $>950 \mu \mathrm{V}$ are shown by diamonds. The theoretical dependence of the linewidth $\Delta f_{\text {LTJ }}$ of a lumped Josephson tunnel junction is ${ }^{11}$

$$
\Delta f_{\mathrm{LTJ}}=\left(2 / \Phi_{0}\right)\left(R_{d}\right)^{2} e I_{\mathrm{dc}} \operatorname{coth}\left[\left(e V_{d c} /\left(2 k_{B} T_{\mathrm{eff}}\right)\right]\right.
$$

where $T_{\text {eff }}$ is the effective temperature of the wide-band noise spectrum. From Fig. 6 one can see that the data obtained for the region with low damping follow the theoretical dependence given by Eq. (2) (dashed line) calculated with an effective temperature $T_{\text {eff }}=27 \mathrm{~K}$, which is about 6.5 times higher than the physical temperature $\left(T_{b}=4.2 \mathrm{~K}\right)$. This is in agreement with previous measurements. ${ }^{6,7}$ At the lowest $R_{d}$ values the external low-frequency noise (interference) seems to be dominant and the dependence $\Delta f\left(R_{d}\right)$ becomes linear:

$$
\Delta f_{\mathrm{lf}}=\left(2 / \Phi_{0}\right) I_{1 \mathrm{ff}} R_{d},
$$

where $I_{\mathrm{lf}}$ is the amplitude of the low-frequency noise current flowing through the junction. The corresponding dependence calculated for $l_{\mathrm{lf}}=0.2 \mu \mathrm{A}$ is shown in Fig. 6 by the dotted line.

For $V>950 \mu \mathrm{V}$ in the "pure" (FS-free) flux-flow regime, the measured linewidths are much higher than found in the "resonant" regime. Furthermore, the $\Delta f\left(R_{d}\right)$ dependence significantly deviates from Eq. (2) even at large $R_{d}$ where the contribution from the low-frequency noise Eq. (3) should be small. Recently, a general model for the FFO linewidth broadening has been proposed and theoretically investigated by Golubov et al. ${ }^{13}$ This model accounts for the fluctuations of the interfluxon spacing in the moving fluxon chain under the influence of noncorrelated spatially distributed thermal noise in the junction. The resulting normalized linewidth is given by the expression

$$
\Delta f_{\mathrm{FF}} / f_{\mathrm{FF}}=4\left[\pi\left(1-v^{2}\right)^{3 / 2} h_{B}^{3}\right]^{-1}\left[\left(L k_{B} T\right) /\left(\lambda_{J} \alpha E_{0}\right)\right]^{1 / 2},
$$

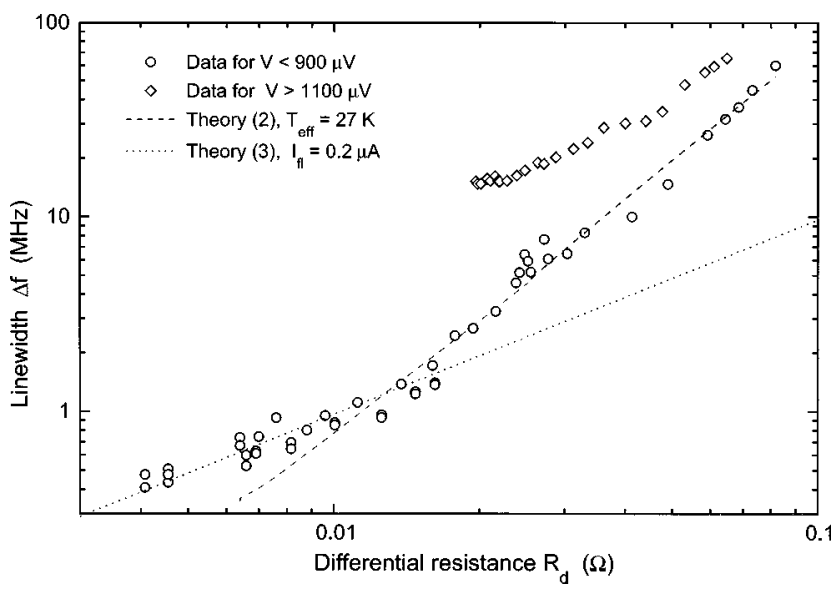

FIG. 6. Dependence of the linewidth $\Delta f$ on the differential resistance $R_{d}$. Data are recorded at $V<900 \mu \mathrm{V}$ (circles) and $V$ $>1100 \mu \mathrm{V}$ (diamonds). Theoretical dependence [Eq. (2)] for the lumped (short) tunnel junction with $T_{\text {eff }}=27 \mathrm{~K}$ is shown by the dashed line; the dependence [Eq. (3)] for a low-frequency noise current $I_{1 \mathrm{f}}=0.2 \mu \mathrm{A}$ is presented by the dotted line. 
where $\nu$ is the fluxon velocity normalized to $c_{\mathrm{Sw}}, h_{B}$ is the normalized magnetic field, and $E_{0}=(4 / \pi) \Phi_{0} j_{c} \lambda_{J} W$ is the Josephson energy. The theoretical estimations ${ }^{13}$ predict a large increase of the FFO linewidth as compared to Eq. (2). This broadening is especially significant at large fluxon velocities and small normalized magnetic field where the fluxon chain is "soft." One can notice that $\Delta f_{\mathrm{FF}}$ diverges as $\nu$ approaches unity, but it should be taken into account that Eq. (4) is derived with the restriction that $\left(1-\nu^{2}\right) \gg 1 / h_{B}^{2}$.

The experimental broadening of the FFO linewidth is much smaller than predicted by Eq. (4). The discrepancy is expected due to the limited range of applicability of the theoretical model. In addition to the restricted velocity range $\left(1-\nu^{2}\right) \gg 1 / h_{B}^{2}$, the model ${ }^{13}$ does not take into account the effect of surface losses which become dominant at high fluxon velocities. For a given $\nu$ the model predicts an increase of the linewidth with decreasing $h_{B}$. This effect, however, is masked in our experiment by the Fiske resonances (which are not accounted for by the theory ${ }^{13}$ ) at low fields.

\section{SUMMARY}

Experimental evidence of FFO self-pumping is presented. We argue that the self-pumping effect strongly influences the $I-V$ characteristics and thus the FFO linewidth. The spectral linewidth has been measured in the frequency range 250$580 \mathrm{GHz}$, and a linewidth as low as $200 \mathrm{kHz}$ is obtained at $450 \mathrm{GHz}$ in the resonant regime with low damping. A significant broadening of the FFO linewidth in the nonresonant flux-flow regime at high voltages is observed. This broadening cannot be adequately described by the existing theoretical models.

\section{ACKNOWLEDGMENTS}

The authors thank A. A. Golubov, E. B. Goldobin, R. Monaco, and R. D. Parmentier for fruitful discussions. The work was supported in part by the Russia Program for Basic Research, the Russian SSP "Superconductivity," INTAS Grant No. 94-1783, the Danish Natural Science Council, and the Danish Research Academy.
${ }^{1}$ T. Nagatsuma, K. Enpuku, F. Irie, and K. Yoshida, J. Appl. Phys. 54, 3302 (1983); see 56, 3284 (1984); 58, 441 (1985); 63, 1130 (1988).

${ }^{2}$ V. P. Koshelets, S. V. Shitov, A. M. Baryshev, I. L. Lapitskaya, L. V. Filippenko, H. van de Stadt, J. Mess, H. Schaeffer, and T. de Graauw, IEEE Trans. Appl. Supercond. 5, 3057 (1995).

${ }^{3}$ V. P. Koshelets, S. V. Shitov, L. V. Filippenko, A. M. Baryshev, H. Golstein, T. de Graauw, W. Luinge, H. Schaeffer, and H. van de Stadt, Appl. Phys. Lett. 68, 1273 (1996).

${ }^{4}$ V. P. Koshelets, S. V. Shitov, A. M. Baryshev, I. L. Lapitskaya, L. V. Filippenko, W. Luinge, H. Golstein, H. van de Stadt, J. R. Gao, and T. de Graauw, IEEE Trans. Appl. Supercond. (to be published).

${ }^{5}$ A. V. Ustinov, T. Doderer, R. P. Huebener, J. Mygind, V. A. Oboznov, and N. F. Pedersen, IEEE Trans. Appl. Supercond. 3, 2287 (1993).

${ }^{6}$ J. Mygind, V. P. Koshelets, A. V. Shchukin, S. V. Shitov, and I. L. Lapitskaya, IEEE Trans. Appl. Supercond. 5, 2951 (1995).

${ }^{7}$ V. P. Koshelets, A. V. Shchukin, I. L. Lapitskaya, and J. Mygind, Phys. Rev. B 51, 6536 (1995).

${ }^{8}$ Y. M. Zhang, D. Winkler, and T. Claeson, Appl. Phys. Lett. 62, 3195 (1993).

${ }^{9}$ A. V. Shchukin, V. P. Koshelets, S. V. Shitov, L. V. Filippenko, and J. Mygind (unpublished).

${ }^{10}$ V. P. Koshelets, S. V. Shitov, A. V. Shchukin, L. V. Filippenko, and J. Mygind, Appl. Phys. Lett. 69, 699 (1996).

${ }^{11}$ M. J. Stephen, Phys. Rev. Lett. 21, 1629 (1968).
${ }^{12}$ E. Jørgensen, V. P. Koshelets, R. Monaco, J. Mygind, M. R. Samuelsen, and M. Salerno, Phys. Rev. Lett. 49, 1093 (1982).

${ }^{13}$ A. A. Golubov, B. A. Malomed, and A. V. Ustinov, Phys. Rev. B 54, 3047 (1996).

${ }^{14}$ M. R. Samuelsen and S. A. Vasenko, J. Appl. Phys. 57, 110 (1985).

${ }^{15}$ V. P. Koshelets, S. V. Shitov, A. V. Shchukin, L. V. Filippenko, I. L. Lapitskaya, and J. Mygind, in Proceedings of the International Conference on Nonlinear Superconducting Devices and HTC Materials, Capri, Italy, 1994, edited by R. D. Parmentier and N. F. Pedersen (World Scientific, Amsterdam, 1995), pp. 383-401.

${ }^{16}$ G. S. Lee, IEEE Trans. Appl. Supercond. 3, 121 (1991); N. Thyssen, A. V. Ustinov, H. Kohlstedt, S. Pagano, J. G. Caputo, and N. Flytzanis, ibid. 5, 2965 (1995).

${ }^{17}$ A. V. Ustinov, H. Kohlstedt, and P. Henne, Phys. Rev. Lett. 77, 3617 (1996).

${ }^{18}$ N. R. Werthamer, Phys. Rev. 147, 255 (1966).

${ }^{19}$ L.-E. Hasselberg, M. T. Levinsen, and M. R. Samuelsen, Phys. Rev. B 9, 3757 (1974).

${ }^{20}$ M. Maezawa, M. Aoyagi, H. Nakagawa, I. Kurosawa, and S. Takada, Phys. Rev. B 50, 9664 (1994).

${ }^{21}$ V. Yu. Belitsky and E. L. Kollberg, J. Appl. Phys. 80, 4741 (1996).

${ }^{22}$ P. M. Marcus and Y. Imry, Solid State Commun. 33, 345 (1980).

${ }^{23}$ N. Grønbech-Jensen, S. A. Hattel, and M. R. Samuelsen, Phys. Rev. B 45, 12457 (1992). 